\title{
Stochastic reconstruction of the microstructure of equilibrium form snow and computation of effective elastic properties
}

\author{
Hongyan YUAN, ${ }^{1}$ Jonah H. LEE, ${ }^{1}$ James E. GUILKEY ${ }^{2}$ \\ ${ }^{1}$ Department of Mechanical Engineering, University of Alaska Fairbanks, Fairbanks, Alaska 99775-5905, USA \\ E-mail: jonah.lee@alaska.edu \\ ${ }^{2}$ Department of Mechanical Engineering, University of Utah, 50 South Central Campus Drive, Salt Lake City, \\ Utah 84112-9208, USA
}

\begin{abstract}
Three-dimensional geometric descriptions of microstructure are indispensable to obtain the structure-property relationships of snow. Because snow is a random heterogeneous material, it is often helpful to construct stochastic geometric models that can be used to model physical and mechanical properties of snow. In the present study, the Gaussian random field-based stochastic reconstruction of the sieved and sintered dry-snow sample with grain size less than $1 \mathrm{~mm}$ is investigated. The one- and two-point correlation functions of the snow samples are used as input for the stochastic snow model. Several statistical descriptors not used as input to the stochastic reconstruction are computed for the real and reconstructed snow to assess the quality of the reconstructed images. For the snow samples and the reconstructed snow microstructure, we also estimate the mechanical properties and the size of the associated representative volume element using numerical simulations as additional assessment of the quality of the reconstructed images. The results indicate that the stochastic reconstruction technique used in this paper is reasonably accurate, robust and highly efficient in numerical computations for the high-density snow samples we consider.
\end{abstract}

\section{INTRODUCTION}

With high-speed large-capacity supercomputing facilities, numerical simulation has become an important practical tool to investigate geophysical or engineering problems involving snow deformation (Bartelt and Lehning, 2002; Schneebeli, 2004; Johnson and Hopkins, 2005). Recently, snow mechanics has been applied to the high-fidelity numerical simulation of tire-snow interaction (Shoop, 2001; Lee, 2009a). However, to obtain reliable results from numerical simulations requires suitable constitutive laws for snow, for the specific problem at hand (small and large deformations, low and high loading rates). This is a challenging task because the macroscopic mechanical behavior depends on the snow microscopic characteristics and other parameters such as temperature and free water content (Johnson, 1998; Johnson and Hopkins, 2005). A comprehensive review of snow mechanics (Shapiro and others, 1997) has pointed out the need to establish an effective snow classification scheme using parameters that reflect microscopic information on snow such that specific macroscopic stress-strain relationships can be ascertained as a function of snow microstructure. Earlier attempts to solve this problem have suffered from the lack of an experimentally based technique to define and measure snow microstructure that allows a correlation to the thermal (Kaempfer and others, 2005) and macroscopic mechanical properties (Brown, 1980; Mahajan and Brown, 1993; Bartelt and Lehning, 2002; Schneebeli, 2004) of snow; additional information can be found using the references cited in this paper.

The microstructural parameters (grain size, bond size, etc.) are generally measured as average values. As a naturally occurring material, snow's microstructure, however, is formed in a stochastic rather than a deterministic sense. Snow then can be regarded as a random porous material, meaning that snow microstructure can also be characterized using statistical descriptors (e.g. two-point correlation function, lineal-path correlation function) to be elaborated in detail below. The effective physical properties of such a random material are determined by the statistical information embedded in the microstructure. The structureproperty relationship for snows has been studied in the past. For example, by assuming the similarity of microstructures between snows and foams, mechanical properties of snows (e.g. Young's modulus) have been modeled based on openand closed-cell foams (Kirchner and others, 2001; Petrovic, 2003). The elastic moduli are the simplest mechanical properties for solids, and hence are studied in this paper for the purpose of evaluating the quality of reconstructed microstructure. Analytical prediction of the upper and lower bounds of the effective elastic moduli of multiphase materials has long been the subject of many theoretical studies (Hashin and Shtrikman, 1963; Hill, 1965; Roberts and Garboczi, 1999; Torquato, 2001); for example, the wellknown Hashin-Shtrikman bounds on elastic moduli (Hashin and Shtrikman, 1963) were obtained knowing only the phase moduli and volume fractions of the material. However, volume fraction is not the only factor determining effective properties. For snow, for example, grain bonds play an important role in determining snow mechanical behaviors (Bartelt and Lehning, 2002; Nicot, 2004; Johnson and Hopkins, 2005). The bounds can be improved by incorporating higher-order correlation functions of the microstructure such that effective properties can be predicted more precisely. On the other hand, large-scale computational methods have proved to be useful in determining composite material properties or behaviors given three-dimensional (3-D) geometric descriptions of the microstructures (Garboczi and Day, 1995; Roberts and Garboczi, 1999; Johnson and Hopkins, 2005). Computational methods can yield direct relationships between the macroscopic properties and the microstructures of multiphase materials, which can 
provide comparison to analytical bounds and experimental results. More importantly, numerical methods, once validated, can provide an alternative method to establish microstructure-property relationships when experimental data are scarce.

The long-term goal of our research program is to establish a classification criterion of the mechanical behavior of snow based on the microstructural statistical information by carrying out large-scale numerical simulations, and compare the results with available experimental data. For microstructure-based numerical analysis, obtaining quantitative geometric representations of snow microstructure is the first step. Recently, X-ray microtomography (XMT) has been utilized to obtain 3-D high-resolution voxel-based images of snow microstructure (Coléou and others, 2001). Experimental methods can be used to obtain real snow microstructure, but conducting the experiments can be demanding. If one can develop a stochastic model of the snow using the microstructural statistics of experimental data as input, it is hoped that one can reduce the number of tests and can obtain microstructure-property relationships using the stochastic model instead. Two-dimensional (2-D) surface micrographs are relatively easy to obtain experimentally. For a statistically isotropic and homogeneous porous material, the porosity and the two-point correlation function calculated from a 2-D image or a 3-D microstructure are the same. Therefore, 3-D microstructure can be numerically simulated by stochastic reconstruction techniques based on the statistical information obtained from 2-D images.

In this paper, the stochastic reconstruction of the snow microstructure and the calculation of effective elastic properties (Young's modulus and Poisson's ratio) are presented. The remainder of this paper is organized as follows. In section 2.1, we review the two widely used stochastic reconstruction methods and introduce in more detail the one chosen for the present study. In section 2.2, we summarize the various quantitative morphological measures that are frequently used in stochastic reconstruction to serve either as target functions or for comparison between the real and simulated microstructures. In section 2.3, we give a brief introduction of the material point method used to simulate the stress and strain fields of representative volume elements (RVEs), and the method of evaluating the effective elastic properties from these fields. In section 3, we describe the acquisition and processing of the real snow microstructure images obtained from XMT. In section 4, we present the results of simulated snow obtained from the stochastic reconstruction and compare them with those of real snow. The conclusion is presented in section 5 .

\section{REVIEW OF THEORY}

\subsection{Stochastic reconstruction}

When snow is considered as a random porous material, its microscopic morphology can be described by a two-valued random field $Z(\mathbf{x})$, which is also referred to as the phase function:

$$
Z(\mathbf{x})= \begin{cases}1, & \text { if } \mathbf{x} \text { belongs to the pore space } \\ 0, & \text { otherwise, }\end{cases}
$$

where $\mathbf{x}$ is the 3-D space vector. The first and second statistical moments of the random field $Z(\mathbf{x})$ are given as

$$
\begin{gathered}
p=\langle Z(\mathbf{x})\rangle, \\
g_{2}(\mathbf{u})=\langle Z(\mathbf{x}) \cdot Z(\mathbf{x}+\mathbf{u})\rangle,
\end{gathered}
$$

where $\mathbf{u}$ is a lag vector and angle brackets denote statistical averages. In this paper, we only consider statistically isotropic and homogeneous porous materials, so $p$ represents the porosity of the porous material, and the correlation function $g_{2}$, which can be written as $g_{2}(u)(u=\|\mathbf{u}\|)$, is only a function of the length of $\mathbf{u}$ and is called the two-point correlation function. The two-point correlation function measures the probability that two points a distance $u$ apart lie in the pore space. For random media without long-range order, it can be proved from Equations (1-3) that $g_{2}(0)=p$, $g_{2}(\infty)=p^{2}$. The two-point correlation function of the solid phase is completely determined by the porosity, and the two-point correlation function of the pore phase by

$$
g_{2}^{\mathrm{s}}(u)=\langle(1-Z(\mathbf{x})) \cdot(1-Z(\mathbf{x}+\mathbf{u}))\rangle=1-2 p+g_{2}(u),
$$

where $g_{2}^{s}(u)$ denotes the two-point correlation function of the solid phase. Therefore, if the two-point correlation function of the pore phase is reproduced in the stochastic reconstruction, that of the solid phase is also reproduced automatically. Moreover, for the reconstruction, only one of them needs to be used as the target information, i.e. the twopoint correlation function cannot distinguish between the two phases.

The purpose of the stochastic reconstruction is to numerically generate realizations of a two-valued random field $Z^{\prime}(\mathbf{x})$ that share the same statistical information (e.g. the porosity and the two-point correlation function) with the real microstructure $Z(\mathbf{x})$. The original motivation for developing stochastic reconstruction methods is that 3-D microstructure of certain materials might be difficult or impossible to obtain experimentally. However, for these materials, 2-D surface micrographs might be readily acquired by conventional microscopy. For a statistically isotropic and homogeneous porous material, certain types of statistical information defined for 2-D surface images are the same as those defined in 3-D space; 3-D microstructure thus can be numerically simulated by stochastic reconstruction techniques based on the statistical information obtained from 2-D images.

Two major algorithms have been developed for the stochastic reconstruction of random media: the Gaussian random field (GRF)-based method (Quiblier, 1984) and the simulated annealing method (Yeong and Torquato, 1998). The GRF-based method is well studied, computationally efficient, but can only reproduce the porosity and the twopoint correlation function of random porous materials. Therefore, it cannot ensure that the higher-order statistical information of the simulated random field is the same as that of the real microstructure. In contrast, the simulated annealing method can include as many statistical constraints as required in the objective function. But as the objective function becomes more complex, the convergence to reach a global minimum is also more difficult to achieve, and much higher computational cost is required.

Because of its high efficiency and robustness (Quintanilla and others, 2007), the GRF-based stochastic reconstruction technique was chosen in this study. Studies of this technique date back to 1974 (Joshi, 1974). Based on Joshi's work, Quiblier (1984) developed the 3-D stochastic reconstruction 
method which has been utilized in many practical problems to provide 3-D microscopic geometric descriptions. These fictitious 3-D microstructures were used to calculate the effective macroscopic properties of random porous or twophase materials (Adler and others, 1990; Kikkinides and others, 2002). Roberts and Teubner (1995) and Roberts (1997) brought this GRF-based stochastic reconstruction method into a general scheme, which can be briefly described as follows.

Let $Y(\mathbf{x})$ denote a real-valued stationary isotropic Gaussian random field that is completely determined by its first and second statistical moments. For simplicity, assume $\langle Y(\mathbf{x})\rangle \equiv 0$ and $\langle Y(\mathbf{x}) \cdot Y(\mathbf{x})\rangle=1$. Many numerical methods have been developed for the generation of realizations of a stationary GRF with a given correlation function $g_{Y}(u)=\langle Y(\mathbf{x}) \cdot Y(\mathbf{x}+\mathbf{u})\rangle$. Therefore, the two-valued random field $Z^{\prime}(\mathbf{x})$ can be simply derived from $Y(\mathbf{x})$ using the following one-level-cut procedure:

$$
Z^{\prime}(\mathbf{x})= \begin{cases}1, & Y(\mathbf{x}) \geq \alpha \\ 0, & Y(\mathbf{x})<\alpha^{\prime}\end{cases}
$$

where $\alpha$ is the one-level-cut parameter. The porosity and the two-point correlation function of $Z^{\prime}(\mathbf{x})$ are denoted by $p^{\prime}=\left\langle Z^{\prime}(\mathbf{x})\right\rangle$ and $g_{2}^{\prime}(u)=\left\langle Z^{\prime}(\mathbf{x}) \cdot Z^{\prime}(\mathbf{x}+\mathbf{u})\right\rangle$, respectively. Thus the remaining task is to ensure that $p^{\prime}=p$ and $g_{2}^{\prime}(u)=g_{2}(u)$. Since $Z^{\prime}(\mathbf{x})$ is associated with $Y(\mathbf{x})$ by Equation (4), there exist relationships between $p^{\prime}, g_{2}^{\prime}(u)$ and $\alpha, g_{\gamma}(u)$ (Teubner, 1991):

$$
\begin{gathered}
p^{\prime}=(2 \pi)^{-1 / 2} \int_{\alpha}^{\infty} \mathrm{e}^{-t^{2} / 2} \mathrm{~d} t \\
g_{2}^{\prime}(u)=p^{\prime}-(2 \pi)^{-1} \int_{g_{Y}(u)}^{1} \frac{1}{\sqrt{1-t^{2}}} \exp \left(-\frac{\alpha^{2}}{1+t}\right) \mathrm{d} t .
\end{gathered}
$$

The statistical reconstruction method adopted in the present study consists of the following steps:

1. $p^{\prime}$ and $g_{2}^{\prime}(u)$ are directly given or computed from 2-D digital images of microstructure,

2. $g_{\gamma}(u)$ and $\alpha$ are computed from $p^{\prime}$ and $g_{2}^{\prime}(u)$ using Equations (5) and (6),

3. with $g_{\gamma}(u)$ known, realizations of GRF $Y(\mathbf{x})$ are numerically generated using the fast Fourier transform (FFT) method that is briefly described below,

4. with the one-level-cut parameter $\alpha$, realizations of $Z^{\prime}(\mathbf{x})$ are generated using Equation (4).

By introducing the one-level-cut process, the original task of generating realizations of two-valued random field $Z^{\prime}(\mathbf{x})$ is then converted into generating realizations of GRF $Y(\mathbf{x})$ with the correlation function $g_{Y}(u)$. An evaluation and comparison of some GRF generators can be found in Fenton (1994). In the present study, the FFT method was adopted to generate realizations of a GRF, as it is efficient and easy to implement. The discrete realizations of the random field $Y(\mathbf{x})$ in a cubic domain $V=L^{3}$ are generated by the $3-\mathrm{D}$ inverse FFT:

$$
\begin{aligned}
& Y_{n_{1} n_{2} n_{3}} \\
& =\frac{1}{N^{3}} \sum_{m_{1}=0}^{N-1} \sum_{m_{2}=0}^{N-1} \sum_{m_{3}=0}^{N-1} \frac{y_{m_{1} m_{2} m_{3}}}{h^{3}} \exp \left(\frac{2 \pi i}{N}\left(m_{1} n_{1}+m_{2} n_{2}+m_{3} n_{3}\right)\right),
\end{aligned}
$$

where $h$ is the sample interval in each direction in the space domain $V$, and $N=L / h$ is the number of sampling points in each direction. Here $y_{m_{1} m_{2} m_{3}}$ are random numbers given by

$$
\begin{array}{r}
y_{m_{1} m_{2} m_{3}}=\left(G_{m_{1} m_{2} m_{3}}^{r e}+i \cdot G_{m_{1} m_{2} m_{3}}^{i m}\right) \\
\cdot \sqrt{\frac{V}{2} \rho\left(\frac{\sqrt{M_{1}^{2}+M_{2}^{2}+M_{3}^{2}}}{L}\right)} \\
M_{j}=\left\{\begin{array}{cc}
m_{j}, & m_{j} \leq N / 2 \\
N-m_{j}, & m_{j}>N / 2, j=1,2,3,
\end{array}\right.
\end{array}
$$

where $G_{m_{1} m_{2} m_{3}}^{r e}$ and $G_{m_{1} m_{2} m_{3}}^{i m}$ are independent Gaussian distributed random variables with zero mean and variance of one, and

$$
\rho(k)=4 \pi \int_{0}^{\infty} g_{Y}(u) u^{2} \frac{\sin (2 \pi k u)}{2 \pi k u} \mathrm{~d} u
$$

is the power spectral density function of the random field $Y(\mathbf{x})$ (Torquato, 2001). It is worth noting that random fields generated by the FFT method automatically possess periodic boundary conditions which are required for statically homogeneous random fields in the sense that the random field is infinitely large.

\subsection{Quantitative measures}

Besides the porosity and the two-point correlation function, there are many other statistical measures that are briefly discussed below. Even though they are not used in the present statistical reconstruction, they can be utilized to evaluate the quality of reconstructed microstructure.

\subsubsection{Specific surface area}

Specific surface area $s$ is defined as the ratio between the inner surface area of a snow sample and its volume. It is indicative of how well the area of the void-solid interface is reproduced. It was shown that the specific surface area for digitized media is a derived quantity of the discrete twopoint correlation function $g_{2}(u)$ (Yeong and Torquato, 1998):

$$
s=-\left.2 D \frac{\mathrm{d} g_{2}(u)}{\mathrm{d} u}\right|_{u=0},
$$

where $D$ is the space dimension. This equation implies that the accuracy of reproducing the specific surface area is closely related to how well the beginning part of the twopoint correlation function is reproduced.

\subsubsection{Correlation length}

The correlation length of the pore space is related to the porosity and the two-point correlation function by (Talukdar and Torsaeter, 2002)

$$
I_{\mathrm{c}}=\int_{0}^{\infty}\left(p-p^{2}\right) g_{2}(u) \mathrm{d} u
$$

Two points with a distance larger than the correlation length are regarded as having no correlation of the value of the phase function and being purely random to each other.

\subsubsection{Lineal path function}

The lineal path function of each phase, defined as $L_{i}(u)$ for statistically isotropic and homogeneous porous materials, is an important morphological quantity that measures the probability of finding a line segment of length $u$ that lies entirely in the ith phase. Unlike the two-point correlation function, the lineal path functions of the pore phase and the solid phase are not simply related to each other. Moreover, 
this function contains some connectedness information that makes it a primary target function in the simulated annealing method (Yeong and Torquato, 1998).

\subsubsection{Chord distribution function}

Chord distribution function of each phase $c_{i}(u)$ is another useful characteristic related to chord length. The quantity $c_{i}(u) \mathrm{d} u$ is defined as the probability that a randomly picked line segment lying entirely in phase $i$ has length in the range from $u$ to $u+\mathrm{d} u$. This quantity directly gives the information about the connectedness and correlation of microstructure.

\subsubsection{Local porosity distribution}

The local porosity distribution $\mu(p, L)$ provides a characteristic length scale for determining the size of the RVE. The quantity $\mu(p, L) \mathrm{d} p$ is the probability that the porosity of a randomly chosen cube of side length $L$ falls into the range $(p, p+\mathrm{d} p)$.

\subsubsection{Pore-size distribution function}

The pore-size distribution function $P(r)$, also referred to as pore-size probability density function, is defined such that $P(r) \mathrm{d} r$ represents the probability that a randomly picked point in the pore phase lies at a distance between $r$ and $r+\mathrm{d} r$ from the nearest point on the pore-solid interface (Torquato, 2001). This function is an intrinsically 3-D microstructural measure and can only be obtained from 3-D samples. Note that if we consider pore phase as one of the two phases, the solid-size distribution function can be defined in the same way as the pore-size distribution function. The algorithm in Bhattacharya and Gubbins (2006) is used in this paper.

\subsection{Effective elastic properties}

In addition to the comparison of quantitative geometric measures discussed above, we assess the quality of reconstructed snow by comparing the effective elastic properties of reconstructed snow with those of real snow via numerical modeling using the material point method (MPM; Sulsky and others, 1994). MPM is one of the meshfree methods that utilize a background mesh as a computational scratch pad. We used the MPM component of the program Uintah, developed at the University of Utah (Parker and others, 2006), mainly due to its parallel capability and the ease of discretizing XMT images into material points (Guilkey and others, 2006). In this paper, each voxel is mapped into a material point. The total number of material points for a typical microstructure is on the order of $50 \times 10^{6}$. A second incentive for using Uintah is to address the more challenging problem of calculating effective visco-plastic properties of snow from XMT images (Lee, 2009b). Like many numerical methods, MPM can use the explicit and implicit algorithms; for this paper, we used the implicit algorithm (Guilkey and Weiss, 2003). Unconfined compression simulation was used to obtain the effective elastic properties; the displacement was prescribed on one side of the cube, and the reaction force was calculated. Effective stress was then calculated by dividing the reaction force by the cross-sectional area; the effective strain was obtained by dividing the displacement by the side length of the snow model. The effective Young's modulus was then calculated by dividing the effective stress by the effective strain. For Poisson's ratio, the average change of the snow model in the lateral direction was first calculated then divided by the side length of the snow model. To obtain statistics of the effective elastic properties, we used the stochastic reconstruction method discussed in this paper to generate five realizations (snow geometries) for reconstructed snow; for real snow, we sampled different parts of the snow to obtain five realizations.

We used two theories to put the results obtained from MPM in perspective. The first is the Hashin-Shtrikman upper-bound bulk modulus $K_{U}$ and shear modulus $G_{U}$ (Hashin and Shtrikman, 1963):

$$
\begin{gathered}
K_{U}=K(1-p)-\frac{p(1-p) K^{2}}{K p+\frac{4}{3} G} \\
G_{U}=G(1-p)-\frac{p(1-p) G^{2}}{G p+H_{2}} \\
H_{2}=G\left(\frac{\frac{3}{2} K+\frac{4}{3} G}{K+2 G}\right),
\end{gathered}
$$

where $p$ is the porosity or the volume fraction of air, and $G$ and $K$ are the shear and bulk modulus of ice, respectively. The second theory we used is for an open-cell foam (Gibson and Ashby, 1997):

$$
E_{\text {snow }}=E_{\text {ice }}\left(\frac{\rho_{\text {snow }}}{\rho_{\text {ice }}}\right)^{2}, \nu_{\text {snow }} \approx \frac{1}{3} .
$$

where $E$ is Young's modulus, $\rho$ is density and $\nu$ is Poisson's ratio. We assume $E_{\text {ice }}=9.3 \mathrm{GPa}$ and $v_{\text {ice }}=0.3$ (Petrenko and Whitworth, 1999).

\section{ANALYSIS OF X-RAY MICROTOMOGRAPHY IMAGES}

The snow sample was collected from the Agricultural Field at the University of Alaska Fairbanks, and subsequently sieved into different grain sizes. The smallest grain-size snow $(<1 \mathrm{~mm})$ was used in this study. Snow samples were put in a custom-made plastic container with a $1 \mathrm{~cm}$ diameter. The plastic container was mounted on the stage of an XMT scanner (SkyScan 1172 microtomograph) located in a cold room at $-15^{\circ} \mathrm{C}$. The scanning duration was from a few minutes to about half an hour, depending on acquisition settings. The exposure time was tuned to prevent snow samples from melting during the course of scanning. A collection of X-ray projection profiles was obtained from the scanner for the cylindrical-shaped snow sample. A series of cross-sectional images were then computed using reconstruction software NRecon. A cross-sectional image of the snow sample is given in Figure 1a. The cross-sectional images were further segmented into binary (black and white) images by computing a histogram of the gray values. Owing to the distinct brightness contrast of the images, the histograms of the images were found to be bimodal (inset in Fig. 1a). Accordingly, the gray value of the valley between two peaks was selected as the segmentation threshold. As shown in Figure 1b, isolated ice phase and pore phase appeared in the segmented images, induced by experimental noise or error of computer reconstruction process; the isolated pixels were removed using CTAn software. The binary counterpart of Figure 1a is shown in Figure 1c, where black pixels represent ice and white pixels denote pore. A 3-D visualization of a cube of the snow sample $3.618 \times 3.618 \times 3.618 \mathrm{~mm}^{3}$ in size is given in Figure $1 \mathrm{~d}$, where shaded area represents ice phase. 


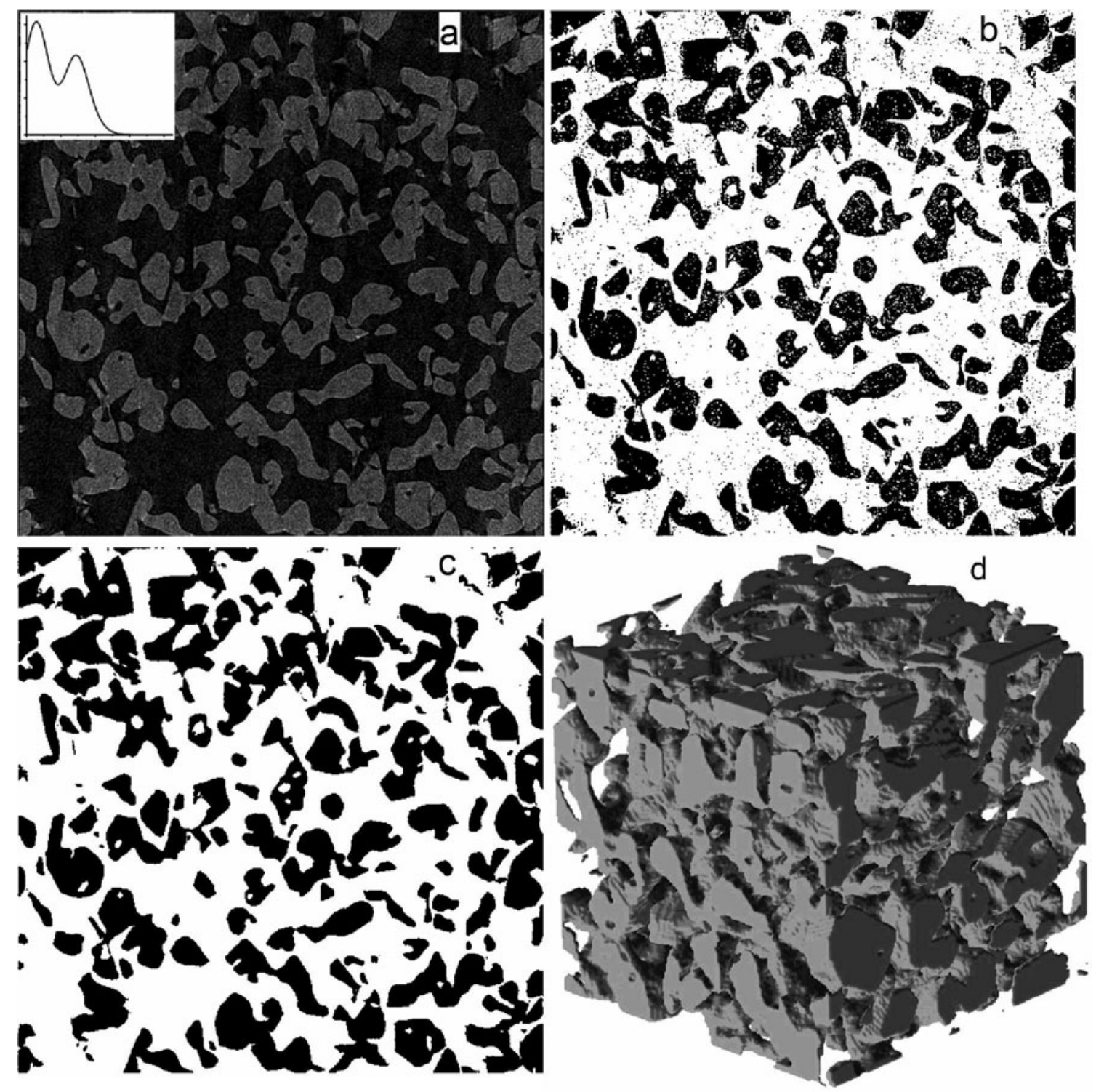

Fig. 1. (a) A gray-level cross-sectional image of the sieved snow sample; image size is $7.35 \times 7.35 \mathrm{~mm}^{2}$ (pixel size $6 \times 6 \mu \mathrm{m}^{2}$ ). (b) The binarized counterpart of (a); small dots of pore and solid phase are artifacts due to the error of the scanning process and reconstruction process. (c) The result of removal of artifacts in (b). (d) 3-D visualization of a cube of snow microstructure, side length $3.618 \mathrm{~mm}$.

The present study is only concerned with statistically isotropic and homogeneous porous materials. Therefore, the statistical average of the random field is identical to the ensemble average. The porosity of the snow sample was computed from the binary images to be 0.585 , implying a snow density of $0.381 \mathrm{~g} \mathrm{~cm}^{-3}$ given the ice density of $0.917 \mathrm{~g} \mathrm{~cm}^{-3}$. The density was also directly estimated by measuring the mass and volume of the snow sample in order to verify the quality of the scanned images and the segmentation procedure. The measured density of the snow sample, $0.377 \mathrm{~g} \mathrm{~cm}^{-3}$, is in good agreement with $0.381 \mathrm{~g}$ $\mathrm{cm}^{-3}$. The two-point correlation function $g_{2}(u)$ of a crosssectional image can be computed using Equation (3) by interpreting the angle bracket as the ensemble average. The two-point correlation function of the snow sample (Fig. 2) depicts the autocorrelation of the microstructure as a function of the lag distance. At zero lag distance, the function is equal to the porosity $p$. At large lag distance, the function approaches its long-range value of $p^{2}$. The statistical isotropy of the snow microstructure is reflected in
Figure 2, in which the two-point correlation functions in three orthogonal directions were found to be almost the same. The correlation length computed using Equation (12) is $56.2 \mu \mathrm{m}$. The porosity and the two-point correlation function given in Figure 2 from the real snow sample are the only input statistical information used for the stochastic reconstruction in the present study.

\section{RESULTS}

With the porosity $p$ and the two-point correlation $g_{2}(u)$ of the real snow sample known, the one-level-cut parameter $\alpha$ and the correlation function $g_{\gamma}(u)$, which are required to generate realizations of the random field, can be obtained by Equations (5) and (6). In the present study, the FFT method was used to numerically generate realizations of the GRF with the given correlation function. The realizations of the two-valued random field $Z^{\prime}(\mathbf{x})$ with desired statistical information were readily obtained through the one-levelcut procedure defined by Equation (4). 


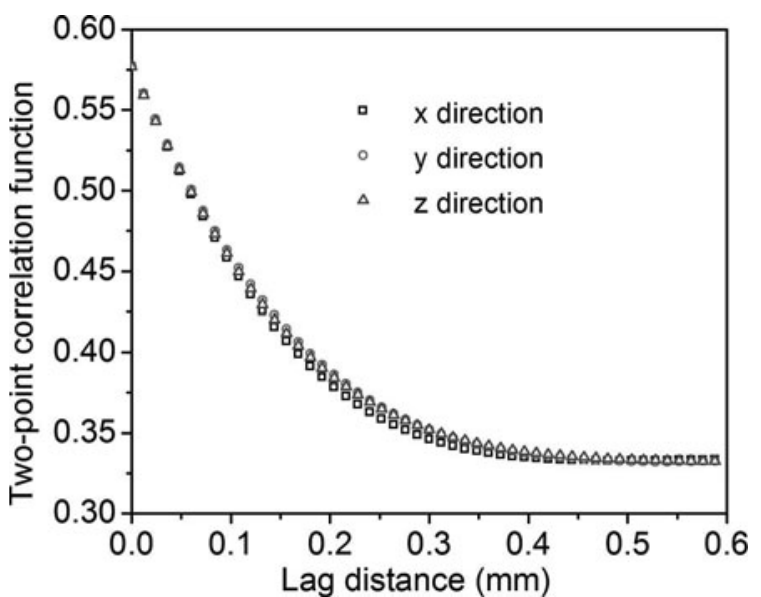

Fig. 2. Two-point correlation functions of the real snow sample in three orthogonal directions. Their consistency implies the statistical isotropy of the sample.

\subsection{Two-dimensional reconstruction results}

Equations (7-10) are written for 3-D cases. Replacing them with their counterparts for 2-D cases in the reconstruction procedure gives rise to the 2-D statistical reconstruction. Two-dimensional stochastic reconstruction can generate much larger images than 3-D reconstruction, computer memory for which is limited. For this reason, large 2-D images were reconstructed for visual evaluation of the reconstruction quality prior to 3-D reconstruction. The simulated 2-D microstructure is given in Figure 3 . The only difference between Figure $3 \mathrm{a}$ and Figure $3 \mathrm{~b}$ is that a highfrequency filter is used in Figure $3 b$ to smooth the pore-solid interface. The threshold of the frequency filter is appropriately tuned such that the porosity change is $<1 \%$. Visual comparison between Figures $3 \mathrm{~b}$ and $1 \mathrm{c}$ indicates that the microstructural morphologies of the simulated and actual images are quite similar. A quantitative comparison between the simulated snow and the real snow sample is presented in section 4.2 .

\subsection{Three-dimensional reconstruction results}

The 3-D reconstruction samples consist of $512 \times 512 \times 512$ voxels (voxel size $12 \times 12 \times 12 \mu \mathrm{m}^{3}$ ). The two-point correlation function of the $3-\mathrm{D}$ simulated microstructure is plotted in Figure 4. It can be seen that the two-point correlation function of the simulated snow matches that of the real snow very well. It is worth noting that the GRF-based reconstruction method is a direct method, whereas the simulated annealing method is an iterative method. Thus, from the algorithmic point of view, the two-point correlation function is precisely reproduced in the GRF-based reconstruction procedure. In practical simulations, simulated finite domain size and domain discretization can result in slight error. The limitation of the GRF-based method is that it cannot ensure that the simulated microstructure has the same higher-order statistical information as the real microstructure.

To assess how well the other statistical information is reproduced, quantitative microstructure descriptors defined in section 2.2 were computed to evaluate the reconstruction quality. The specific surface area and correlation length are listed in Table 1. The reconstructed snow has a slightly higher specific surface area and correlation length than the real snow. a

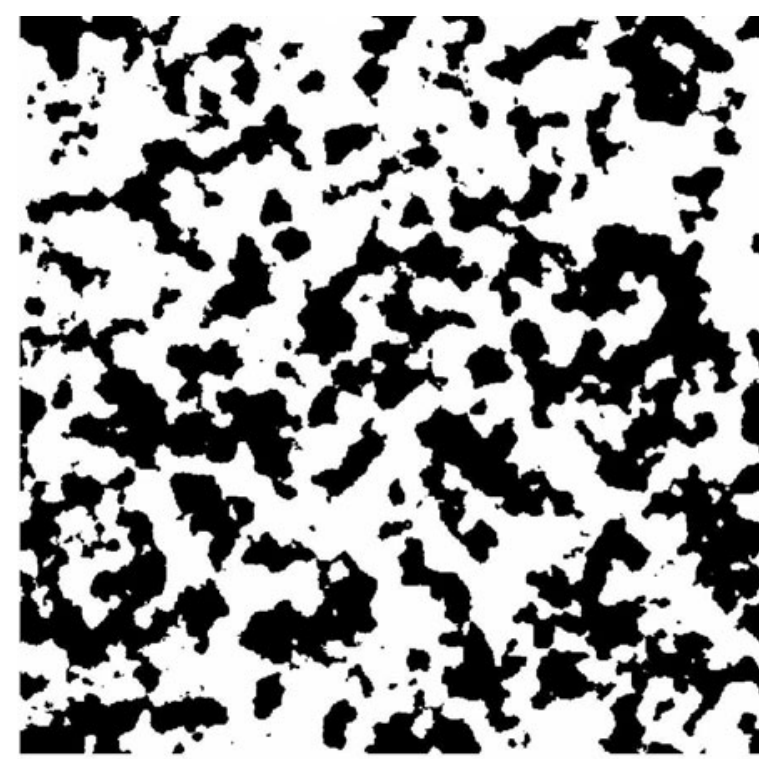

b

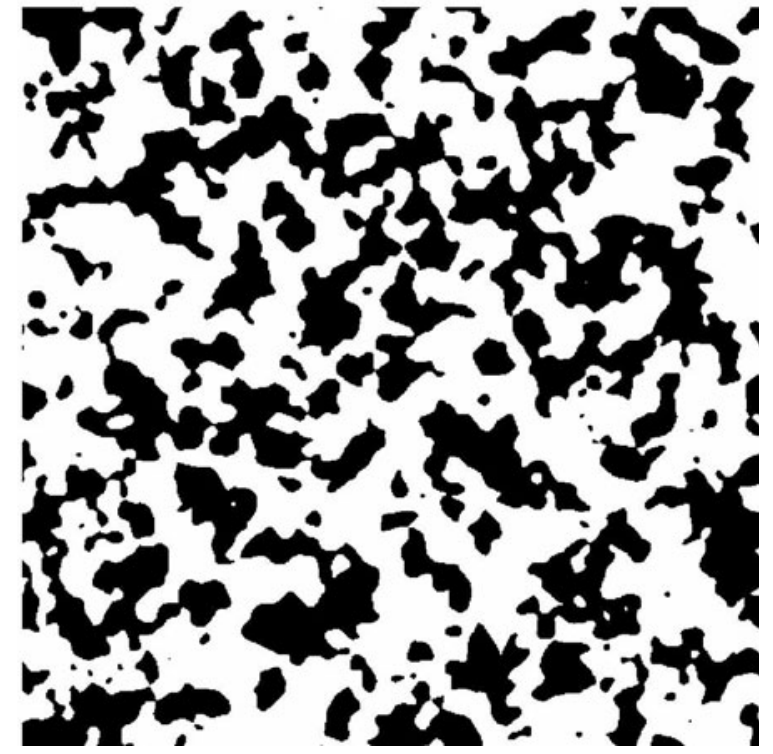

Fig. 3. The simulated 2-D microstructure; image size is $7.35 \times 7.35 \mathrm{~mm}^{2}$ (pixel size $6 \times 6 \mu \mathrm{m}^{2}$ ). (a) Without high-frequency filtering. (b) High-frequency components filtered out to make the pore-solid interface smoother.

The comparison of the lineal path functions is given in Figure $5 \mathrm{a}$ and $\mathrm{b}$. It is important to stress that the lineal path function is reasonably well reproduced even though it is not used in the reconstruction. A similar conclusion was made in a study of the reconstruction of chalk pore networks using the simulated annealing method (Talukdar and Torsaeter, 2002) where it was found that imposing the lineal path function as the target function in addition to the two-point correlation function only improves the reconstruction quality slightly. The drawback of the GRF-based reconstruction is that it only reproduces the first- and second-order statistical information. However, if the medium to be reconstructed is close to the Gaussian distribution, then no higher statistical information is needed and reasonable accuracy can be expected. Figure $5 \mathrm{a}$, however, does show that the solid-phase lineal path function is slightly higher for the reconstructed than for the real snow, indicating that the solid phase of the reconstructed snow is more connected than that of the real snow. This also implies that the pore 


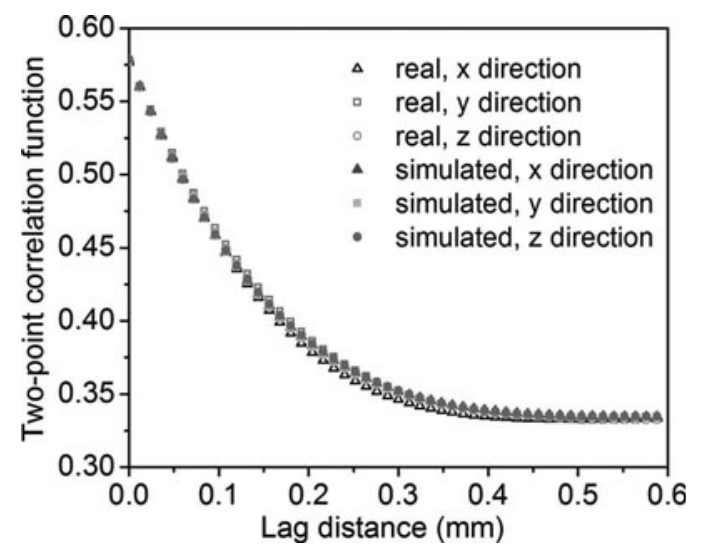

Fig. 4. The two-point correlation function of real and simulated snow. The two-point correlation function of the simulated snow matches well with that of the real snow.
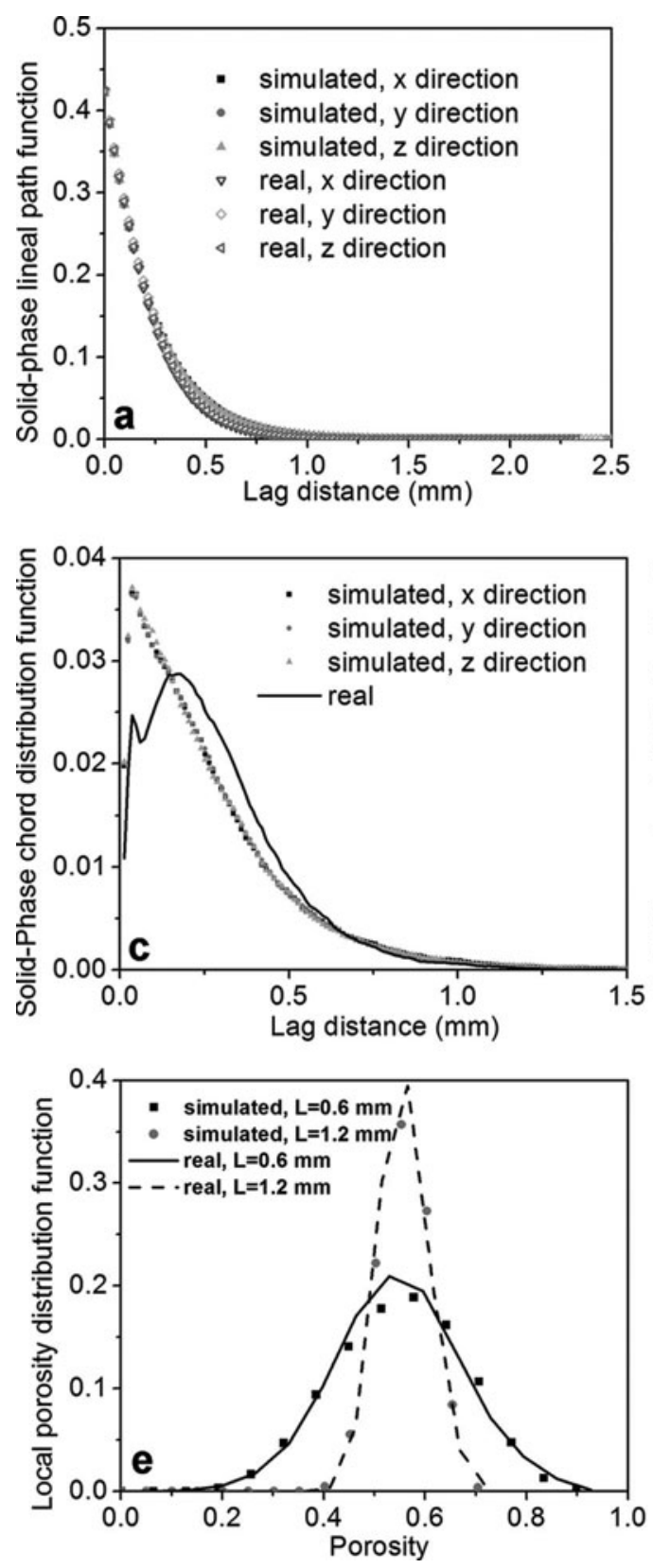

Table 1. Summary of reconstruction results $(512 \times 512 \times 512$ voxels)

\begin{tabular}{lcc}
\hline Parameter & Simulated snow & Real snow \\
\hline Specific surface area, $s\left(\mathrm{~mm}^{-1}\right)$ & 9.1 & 8.6 \\
Correlation length, $I_{\mathrm{c}}(\mu \mathrm{m})$ & 57.8 & 56.2 \\
\hline
\end{tabular}

phase of the reconstructed snow is less connected than that of the real snow, as shown in Figure 5b. We attribute this discrepancy to the nature of the Gaussian distribution and the absence of higher statistical information as inputs. Incorporation of other statistical information will be attempted in future work.
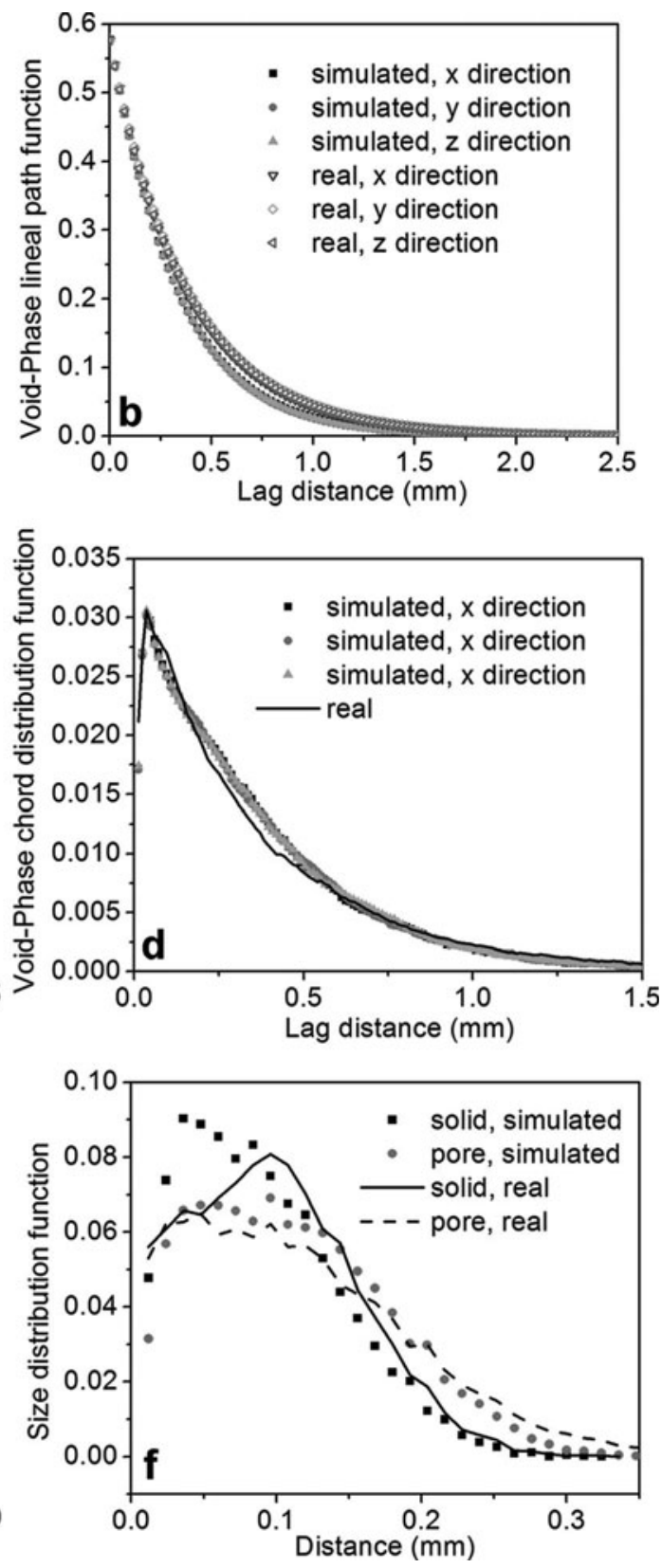

Fig. 5. Quantitative comparisons of the microstructure descriptors: (a) solid-phase lineal path functions; (b) void-phase lineal path functions; (c) solid-phase chord distribution functions; (d) void-phase chord distribution functions; (e) local porosity distribution functions; and (f) poresize and solid-size distribution functions. 


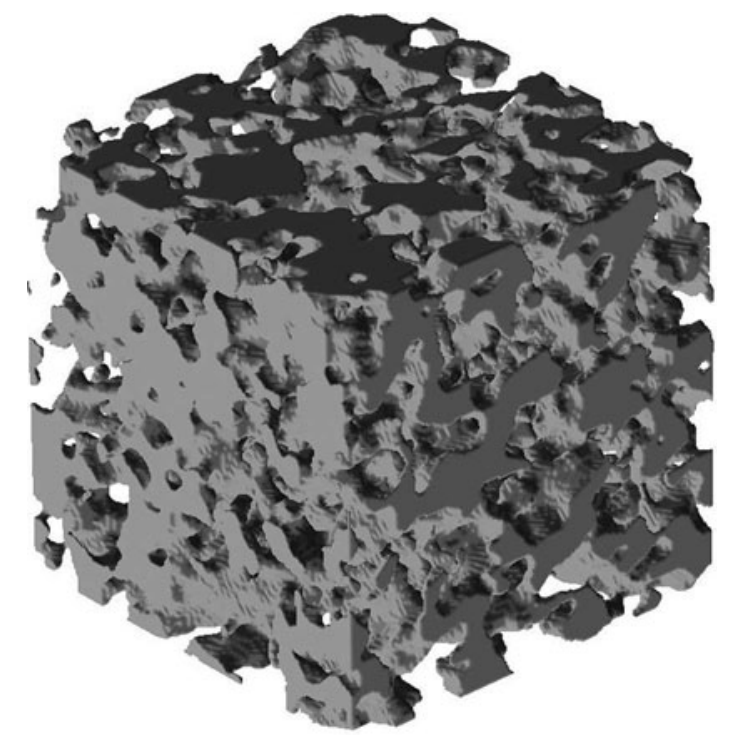

Fig. 6. 3-D visualization of the simulated snow microstructure $\left(3.84 \times 3.84 \times 3.84 \mathrm{~mm}^{3}\right)$.

A reasonable match between simulated and real snow was also found for the other functions shown in Figure $5 c-f$. The void-phase chord distribution function is well reproduced as shown in Figure $5 d$. However, the peaks of the solid-phase chord distributions shown in Figure 5c vary significantly, indicating there are more single-voxel solidphase chords for the reconstructed snow.

The local porosity distribution functions and the size distribution functions are compared in Figure $5 \mathrm{e}$ and $\mathrm{f}$, respectively. The solid and pore-size distribution functions (Fig. 5f) are similar to the solid- and void-phase chord distribution functions. A 3-D visualization of a cube of the simulated sample $3.84 \times 3.84 \times 3.84 \mathrm{~mm}^{3}$ in size is shown in Figure 6 where shaded area represents the solid phase.

\subsection{Results of elastic moduli}

Figure 7 shows the comparison of Young's moduli and Poisson's ratios (in means and standard deviations) for real and reconstructed snow as a function of the size of the snow cube using MPM simulations. For the largest real snow of $7.488 \mathrm{~mm}$ side length, only one sample is available, so no statistics can be obtained. For real snow, the Young's modulus remains almost constant, but increases slightly with side length. For reconstructed snow, no trend can be observed. Table 2 shows that the reconstructed snow has a higher Young's modulus than the real snow; this is attributed to the higher connectedness of the solid phase of the reconstructed snow (cf. Fig. 5a, c and f). The Young's moduli for both real and reconstructed snows are at the higher end of the test data, probably because the snow used in this paper is sieved and more uniform. Since ice creeps easily, it is difficult to measure the Young's modulus of snow accurately by isolating the viscous effect (Kirchner and others, 2001), i.e. some test results of Young's modulus reported in the literature could be a tangent modulus which is smaller than the intrinsic Young's modulus, such as calculated in this paper, where viscous effect is absent in the constitutive model; our approach is similar to that of Schneebeli (2004) where the order of magnitude of Young's modulus calculated is similar to ours. The Young's moduli from simulations are all much smaller than those due to the open-cell foam model or the theoretical Hashin-Shtrikman bound; the moduli from open-cell foam can thus be considered a better upper bound than the Hashin-Shtrikman bound for the snow studied. The Poisson's ratios are all smaller than those from the test data and theories. It is known that Poisson's ratio is more difficult to ascertain for a porous than for a solid material (Gibson and Ashby, 1997); this aspect needs further investigation. Although it is difficult to draw a definitive conclusion regarding the size of the RVE for the snow being studied, 5-6 $\mathrm{mm}$ seems to be a reasonable range judging from the distribution of Young's moduli in Figure 7.

\section{CONCLUSIONS}

Obtaining a quantitative geometric description of snow microstructure is essential to investigations of microstructure-based mechanical or thermal behaviors of snow. Despite recent advances in experimental methods (e.g. $\mathrm{XMT}$ ), stochastic reconstruction of snow microstructure based on statistical modeling, being a numerical method, can be an important alternative. The GRF-based stochastic reconstruction of the sieved and sintered dry-snow sample with grain size $<1 \mathrm{~mm}$ has been investigated in the present study. The visual and quantitative comparisons between the simulated and real snow microstructure indicated that the stochastic reconstruction of this type of snow microstructure
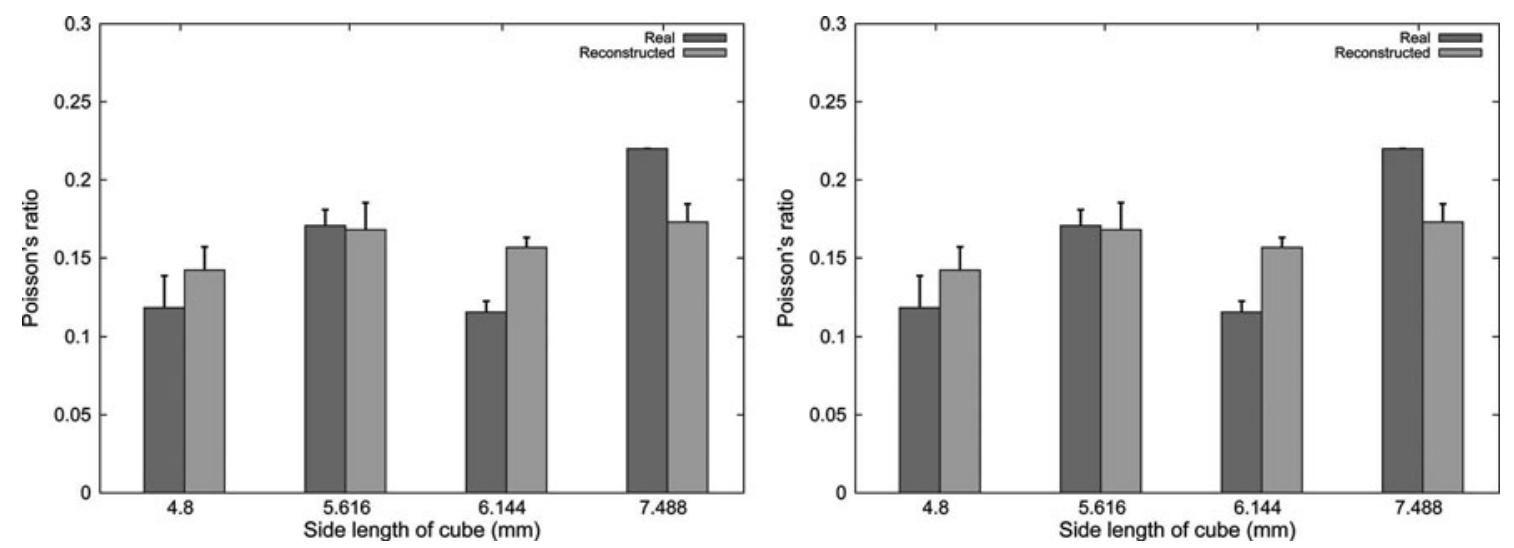

Fig. 7. Means and standard deviations of elastic moduli (a) and Poisson's ratios (b) of simulated and real snow as a function of the side length of the snow. 
Table 2. Summary of elastic moduli from simulations and theories

Young's modulus, $E$ Poisson's ratio, $\nu$ $\mathrm{GPa}$

$\begin{array}{lcc}\text { Reconstructed snow sample } & 0.694 \pm 0.056 & 0.152 \pm 0.018 \\ \text { Real snow sample } & 0.581 \pm 0.044 & 0.153 \pm 0.044 \\ \text { Test data (Shapiro and others, } & & \\ \text { 1997) } & 0.01-0.8 & 0.22-0.35 \\ \text { Open-cell foam } & 1.72 & 0.33 \\ \text { Hashin-Shtrikman } & 2.49 & 0.27\end{array}$

is reasonably accurate even though only the porosity and the two-point correlation function were used. The nature of Gaussian distribution and the absence of higher statistical information as inputs may lead to the higher connectedness in the simulated than in the real microstructure. The drawback of the GRF-based reconstruction is that it only reproduces first- and second-order statistical information. However, if the medium to be reconstructed is close to the Gaussian distribution, then higher statistical information is not needed and reasonable accuracy can be expected. In conclusion, the GRF-based stochastic reconstruction technique is reasonably accurate, robust and highly efficient in numerical simulations, making it a promising algorithm for providing 3-D quantitative geometric descriptions of random porous materials.

The preliminary computations of the effective elastic moduli of varying sizes of RVEs demonstrated the potential of the stochastic modeling of snow microstructure for investigating the microstructure-property relationship. Further work is needed to determine this relationship by obtaining improved statistical information for the stochastic reconstruction beyond the porosity and the two-point correlation function.

\section{ACKNOWLEDGEMENTS}

We thank H. Löwe for helpful comments on the manuscript. This work was supported in part by a grant of highperformance computing resources from the Arctic Region Supercomputing Center at the University of Alaska Fairbanks as part of the US Department of Defense High Performance Computing Modernization Program. J.H. Lee and H. Yuan gratefully acknowledge financial support from the Automotive Research Center (ARC), a US Army Research, Development and Engineering Command (RDECOM) Center of Excellence for Modeling and Simulation of Ground Vehicles led by the University of Michigan, and the support of the US Army Tank Automotive Research, Development and Engineering Center (TARDEC). J.E. Guilkey gratefully acknowledges financial support from DOE W-405-ENG-48 (Center for the Simulation of Accidental Fires and Explosions (C-SAFE), University of Utah).

\section{REFERENCES}

Adler, P.M., C.G. Jacquin and J.A. Quiblier. 1990. Flow in simulated porous-media. Int. J. Multiphase Flow, 16(4), 691-712.

Bartelt, P. and M. Lehning. 2002. A physical SNOWPACK model for the Swiss avalanche warning. Part I: numerical model. Cold Reg. Sci. Technol., 35(3), 123-145.
Bhattacharya, S. and K.E. Gubbins. 2006. Fast method for computing pore size distributions of model materials. Langmuir, 22(18), 7726-7731.

Brown, R.L. 1980. A volumetric constitutive law for snow based on a neck growth model. J. Appl. Phys., 51(1), 161-165.

Coléou, C., B. Lesaffre, J.B. Brzoska, W. Ludwig and E. Boller. 2001. Three-dimensional snow images by $\mathrm{X}$-ray microtomography. Ann. Glaciol., 32, 75-81.

Fenton, G.A. 1994. Error evaluation of three random-field generators. J. Eng. Mech., 120(12), 2478-2497.

Garboczi, E.J. and A.R. Day. 1995. An algorithm for computing the effective linear elastic properties of heterogeneous materials: three-dimensional results for composites with equal phase Poisson ratios. J. Mech. Phys. Solids, 43(9), 1349-1362.

Gibson, L.J. and M.F. Ashby. 1997. Cellular solids: structure and properties. Second edition. Cambridge, etc., Cambridge University Press.

Guilkey, J.E. and J.A. Weiss. 2003. Implicit time integration for the material point method: quantitative and algorithmic comparisons with the finite element method. Int. J. Num. Meth. Eng., 57(9), 1323-1338.

Guilkey, J.E., J.B. Hoying and J.A. Weiss. 2006. Computational modeling of multicellular constructs with the material point method. J. Biomech., 39(11), 2074-2086.

Hashin, Z. and S. Shtrikman. 1963. A variational approach to the theory of the elastic behaviour of multiphase materials. J. Mech. Phys. Solids, 11(2), 127-140.

Hill, R. 1965. A self-consistent mechanics of composite materials. J. Mech. Phys. Solids, 13(4), 213-222.

Johnson, J.B. 1998. A preliminary numerical investigation of the micromechanics of snow compaction. Ann. Glaciol., 26, 51-54.

Johnson, J.B. and M.A. Hopkins. 2005. Identifying microstructural deformation mechanisms in snow using discrete-element modeling. J. Glaciol., 51(173), 432-442.

Joshi, M.Y. 1974. A class of stochastic models for porous media. (PhD thesis, University of Kansas.)

Kaempfer, T.U., M. Schneebeli and S.A. Sokratov. 2005. A microstructural approach to model heat transfer in snow. Geophys. Res. Lett., 32(21), L21503. (10.1029/2005GL023873.)

Kikkinides, E.S., K.L. Stefanopoulos, T.A. Steriotis, A.C. Mitropoulos, N.K. Kanellopoulos and W. Treimer. 2002. Combination of SANS and 3-D stochastic reconstruction techniques for the study of nanostructured materials. Appl. Phys. A., 74(1), S954-S956.

Kirchner, H.O.K., G. Michot, H. Narita and T. Suzuki. 2001. Snow as a foam of ice: plasticity, fracture and the brittle-to-ductile transition. Philos. Mag. A, 81(9), 2161-2181.

Lee, J.H. 2009a. A new indentation model for snow. J. Terramech., 46(1), 1-13.

Lee, J.H. 2009b. Plane strain indentation of snow at the microscale. In Proceedings of the 2008 ASME International Design Engineering Technical Conference: 10th International Conference on Advanced Vehicle and Tire Techologies, 3-6 August 2008, Brooklyn, New York. Fairfield, NJ, American Society of Mechanical Engineers.

Mahajan, P. and R.L. Brown. 1993. A microstructure-based constitutive law for snow. Ann. Glaciol., 18, 287-294.

Nicot, F. 2004. Constitutive modelling of snow as a cohesivegranular material. Granular Matter, 6(1), 47-60.

Parker, S.G., J. Guilkey and T. Harman. 2006. A component-based parallel infrastructure for the simulation of fluid-structure interaction. Eng. Comput., 22(3-4), 277-292.

Petrenko, V.F. and R.W. Whitworth. 1999. Physics of ice. Oxford, etc., Oxford University Press.

Petrovic, J.J. 2003. Review: mechanical properties of ice and snow. J. Mater. Sci., 38(1), 1-6.

Quiblier, J.A. 1984. A new three-dimensional modeling technique for studying porous media. J. Colloid Interface Sci., 98(1), 84-102.

Quintanilla, J.A., J.T. Chen, R.F. Reidy and A.J. Allen. 2007. Versatility and robustness of Gaussian random fields for model- 
ling random media. Model. Simulat. Mater. Sci. Eng., 15(4), S337-S351.

Roberts, A.P. 1997. Statistical reconstruction of three-dimensional porous media from two-dimensional images. Phys. Rev. E, 56(3), 3203-3212.

Roberts, A.P. and E.J. Garboczi. 1999. Elastic properties of a tungsten-silver composite by reconstruction and computation. J. Mech. Phys. Solids, 47(10), 2029-2055.

Roberts, A.P. and M. Teubner. 1995. Transport properties of heterogeneous materials derived from Gaussian random fields: bounds and simulation. Phys. Rev. E, 51(5), 4141-4154.

Schneebeli, M. 2004. Numerical simulation of elastic stress in the microstructure of snow. Ann. Glaciol., 38, 339-342.

Shapiro, L.H., J.B. Johnson, M. Sturm and G.L. Blaisdell. 1997. Snow mechanics: review of the state of knowledge and applications. CRREL Rep. 97-3.
Shoop, S.A. 2001. Finite element modeling of tire-terrain interaction. CRREL Tech. Rep. 01-16.

Sulsky, D., Z. Chen and H.L. Schreyer. 1994. A particle method for history-dependent materials. Comput. Meth. Appl. Mech. Eng., 118(1-2), 179-196.

Talukdar, M.S. and O. Torsaeter. 2002. Reconstruction of chalk pore networks from 2-D backscatter electron micrographs using a simulated annealing technique. J. Petrol. Sci. Eng., 33(4), 265-282.

Teubner, M. 1991. Level surfaces of Gaussian random fields and microemulsions. Europhys. Lett., 14(5), 403-408.

Torquato, S. 2001. Random heterogeneous materials: microstructure and macroscopic properties. New York, SpringerVerlag.

Yeong, C.L.Y. and S. Torquato. 1998. Reconstructing random media. Phys. Rev. E, 57(1), 495-506.

MS received 1 November 2009 and accepted in revised form 13 April 2010 